\title{
Adding Insult to Injury: Mitigating the Impact of COVID-19 on Mental Health Treatment of PTSD
}

\author{
Greg M. Reger ${ }^{1}$ (D) Barbara O. Rothbaum ${ }^{2}$ \\ Received: 19 June 2020 / Revised: 19 June 2020 / Accepted: 31 August 2020 / Published online: 11 September 2020 \\ (C) This is a U.S. government work and not under copyright protection in the U.S.; foreign copyright protection may apply 2020
}

Tremendous progress has been made in the dissemination of recommended, first-line psychotherapies for PTSD among military service members and veterans (Karlin and Cross 2014). However, recent public health infection prevention interventions for COVID-19 require widespread adoption of social distancing to slow the spread of the virus. These interventions risk negative impacts on the mental health treatment of posttraumatic stress disorder (PTSD). Technology has an important role to play in supporting outcomes during this era, particularly should fears of a "2nd wave" of COVID-19 infection come to pass.

\section{Impact of the Public Health Management of Novel Corona Virus on PTSD Treatment}

The most direct impact of the spread of COVID-19 on PTSD treatment is the reduced access to treatment as the virus spreads. Non-urgent healthcare encounters are being delayed or canceled in many parts of the country. Intensive groupbased psychotherapy has ceased in some regions. This involuntary break in treatment for those engaged in PTSD therapy is concerning and the impact is not known. Recent data suggest lower attrition and better outcomes for evidence-based psychotherapy for PTSD among those with more frequent appointments, or intensive treatment (Foa et al. 2018).

Social distancing also risks reinforcing PTSD-related avoidance. Theories of PTSD conceptualize patients' attempts to manage distress through avoidance as maladaptive, as it prevents the learning essential to recovery (Foa et al. 1989).

Greg M. Reger

Greg.reger@va.gov

1 VA Puget Sound Health Care System, 9600 Veterans Drive, A-116, Tacoma, WA 98493, USA

2 Emory School of Medicine, 12 Executive Park Drive, Atlanta, GA 30329, USA
Exposure therapies are highly effective treatments but involve patient collaboration to block avoidance and intentionally approach previously avoided memories, situations, and places. Current social distancing directly interferes with treatment via in vivo exposure, as many patients can no longer approach common treatment assignments like crowds, some stores, and public environments.

As the health crisis continues, the anxiety of the general population understandably increases with a near-constant consumption of news, which focuses attention on the many risks and concerns of the day. With "stay at home" requirements in a number of states, patients engaged in PTSD treatment have limited access to many of their traditional forms of coping (e.g., gyms, faith communities, social gatherings).

\section{PTSD Treatment Opportunities During Pandemic}

To support patients with PTSD at this time, providers should consider adoption of telemental health modalities to reduce treatment interruptions. Evidence-based treatments for PTSD delivered via telehealth are equivalent to face-to-face care (Morland et al. 2019) and the U.S. Department of Health and Human Services has waived potential penalties for HIPAA violations during care of patients via communication applications like Skype or Face Time (U.S. Department of Health and Human Service 2020). While careful attention to ethical practice (Barnett in press) and clinician telebehavioral health competencies (Maheu et al. 2018) must persist, telemental health provides opportunities to maintain continuity of care throughout the pandemic.

Virtual reality (VR) technology gives the user the psychological sense of participating in a computer-generated environment and VR stimuli have demonstrated equivalence with realworld in vivo exposure for some generalized fear stimuli (Carl et al. 2019). VR or other technology solutions can be considered to fill the void during limited in vivo exposure opportunities in social or public settings. If patients have limited access to 
VR interventions, free online virtual worlds, such as Second Life, might be examined for relevance. Clinicians can also consider creative solutions for exposure, such as finding and assigning YouTube video content relevant to in vivo goals.

Clinicians need to support innovative solutions for behavioral activation that do not involve physical social gatherings and public spaces. Social media may help fill some gaps and home-based hobbies may receive increased attention. Leaving the home for walks and activity should be encouraged and scheduled. When that is not feasible, virtual behavioral activation can be considered through internet resources. Patients with PTSD are at risk for maladaptive avoidance and the COVID-19 pandemic has created a socially condoned reason to persist with maladaptive patterns. Technology can help clinicians address overgeneralizations of "stay at home" requirements to ensure that adaptive exposure opportunities that do exist are not avoided.

\section{References}

Barnett, J. E. (in press). Ethical and legal issues in the clinical use of technology. In G. M. Reger (Ed.), Technology and mental health: a clinician's guide to improving outcomes. Routledge.

Carl, E., Stein, A. T., Levihn-Coon, A., Pogue, J. R., Rothbaum, B., Emmelkamp, P., Asmundson, G. J. G., Carlbring, P., \& Powers, M. B. (2019). Virtual reality exposure therapy for anxiety and relat- ed disorders: a meta-analysis of randomized controlled trials. Journal of Anxiety Disorders, 61, 27-36.

Foa, E. B., Steketee, G., \& Rothbaum, B. O. (1989). Behavioral/cognitive conceptualizations of post-traumatic stress disorder. Behavior Therapy, 20, 155-176.

Foa, E. B., McLean, C. P., Zang, Y., Rosenfield, D., Yadin, E., Yarvis, J. S., Mintz, J., Young-McCaughan, S., Borah, E. V., Dondanville, K. A., Fina, B. A., Hall-Clark, B. N., Lichner, T., Litz, B. T., Roache, J., Wright, E. C., \& Peterson, A. L. (2018). Effect of prolonged exposure therapy delivered over 2 weeks vs 8 weeks vs presentcentered therapy on PTSD symptom severity in military personnel: a randomized clinical trial. JAMA, 319, 354-364.

Karlin, B. E., \& Cross, G. (2014). From the laboratory to the therapy room: national dissemination and implementation of evidencebased psychotherapies in the US Department of Veterans Affairs Health Care System. American Psychologist, 69, 19-33.

Maheu, M., Drude, K., Hertlein, K., \& Hilty, D. (2018). A framework of interprofessional telebehavioral health competencies: implementation challenges moving forward. Academic Psychiatry, 42(6), 824-833.

Morland, L. A., Mackintosh, M. A., Glassman, L. H., Wells, S. Y., Thorp, S. R., Rauch, S. A. M., Cunningham, P. B., Tuerk, P. W., Grubbs, K. M., Golshan, S., Sohn, M. J., \& Acierno, R. (2019). Home-based delivery of variable length prolonged exposure therapy: a comparison of clinical efficacy between service modalities. Depression \& Anxiety.

U.S. Department of Health \& Human Services. (2020, March 30). Notification of enforcement discretion for telehealth remote communications during the COVID-19 nationwide public health emergency. https:/www.hhs.gov/hipaa/for-professionals/special-topics/ emergency-preparedness/notification-enforcement-discretiontelehealth/index.html. Accessed 8 Sept 2020.

Publisher's Note Springer Nature remains neutral with regard to jurisdictional claims in published maps and institutional affiliations. 